\title{
A NOTE ON THE CONGRUENCE LATTICE OF A FINITELY GENERATED ALGEBRA ${ }^{1}$
}

\author{
IVAN RIVAL AND BILL SANDS
}

\begin{abstract}
Let $\mathfrak{A}$ be a finitely generated algebra of finite type. If $\theta$ is a congruence relation of $\mathfrak{A}$ such that $\mathfrak{A} / \theta$ is finite then $\theta$ is compact in the lattice $\operatorname{Con}(\mathfrak{A})$ of all congruence relations of $\mathfrak{A}$. Moreover, if $\mathfrak{A}$ is infinite then there is a congruence relation $\boldsymbol{\theta}$ such that $\mathfrak{A} / \boldsymbol{\theta}$ is infinite and $\mathfrak{X} / \boldsymbol{\theta}^{\prime}$ is finite for every $\theta^{\prime}>\theta$ in $\operatorname{Con}(\mathfrak{U})$.
\end{abstract}

The study of finitely generated algebras is important to the theory of algebraic systems. Problems concerning free algebras and varieties of algebras often turn on this theme. At the same time properties of the lattice of congruence relations of an algebra often bear immediate consequences for the structure theory of an algebraic system.

Let $\mathfrak{A}$ be an algebra, that is, a pair $\langle A, F\rangle$, where $A$ is a set and $F$ is a family of operations on $A$. We say that $\mathfrak{A}$ is of finite type if $|F|$ is finite. Let Con $(\mathfrak{A})$ denote the lattice of all congruence relations of $\mathfrak{A}$ partially ordered by set inclusion. For elements $x$ and $y$ of $A$ let $\theta(x, y)$ denote the principal congruence relation generated by identifying $x$ and $y$; that is, $\theta(x, y)=$ $\bigwedge(\theta \mid \theta \in \operatorname{Con}(\mathfrak{A})$ and $x \equiv y(\theta))$. For a general reference to terminology we refer the reader to [1] or [3].

THEOREM 1. ${ }^{2}$ Let $\mathfrak{A}$ be a finitely generated algebra of finite type and let $\theta$ be $a$ congruence relation of $\mathfrak{A}$. If $\mathfrak{A} / \boldsymbol{\theta}$ is finite then $\theta$ is compact in Con( $\mathfrak{U})$.

AN INTERPRETATION. Because a compact congruence relation is simply a join of finitely many principal congruence relations, and because any element of $A$ is a polynomial expression in the generators of $A$, the Theorem states that a finite homomorphic image of a finitely generated algebra can be obtained by imposing finitely many polynomial relations on the generators.

Proof. For subsets $B$ and $C$ of $A$ set

$$
\theta(B, C)=\bigvee(\theta(b, c) \mid b \in B, c \in C \text {, and } \theta(b, c) \leqslant \theta) .
$$

Let $G_{0}$ be a finite subset of $A$ which generates $\mathfrak{A}$ and, for each integer $n \geqslant 1$ set

$$
G_{n}=\left\{f\left(a_{1}, a_{2}, \ldots, a_{m}\right) \mid f \in F \text { and } a_{1}, a_{2}, \ldots, a_{m} \in G_{n-1}\right\} \cup G_{n-1} .
$$

Received by the editors August 19, 1977 and, in revised form, November 14, 1977. AMS (MOS) subject classifications (1970). Primary 08A05; Secondary 06A35.

${ }^{1}$ This work was supported in part by N.R.C. Grant No. A4077.

${ }^{2}$ The authors are grateful to Kirby Baker, Ralph Freese, and George Grätzer for suggesting the present scope and form of this theorem. An earlier draft of this note had developed an equivalent theory for lattices only, in terms of ideals in their congruence lattices. 
Observe that

$$
\bigcup\left(G_{n} \mid n=0,1,2, \ldots\right)=A
$$

We define the rank $r(x)$ of an element $x \in A$ as the least integer $n$ such that $x \in G_{n}$. Let $S_{0}$ be a system of representatives of the $\theta$-classes of $A$ and set

$$
S_{1}=\left\{f\left(s_{1}, s_{2}, \ldots, s_{m}\right) \mid f \in F \text { and } s_{1}, s_{2}, \ldots, s_{m} \in S_{0}\right\} .
$$

Let $\theta^{\prime}=\theta\left(S_{0}, S_{1}\right) \vee \theta\left(S_{0}, G_{0}\right)$. Evidently, $\theta^{\prime} \leqslant \theta$ and, as $\theta^{\prime}$ is a finite join of principal congruence relations of $\mathfrak{A}$, it is compact in $\operatorname{Con}(\mathfrak{U})$. We shall show that $\theta=\theta^{\prime}$, by proving that $S_{0}$ is also a set of representatives for the $\theta^{\prime}$-classes of $\boldsymbol{A}$.

We must prove that, for every $x \in A, x \equiv s\left(\theta^{\prime}\right)$ for some $s \in S_{0}$. We do this by induction on $r(x)$. If $x \in G_{0}$ then $x \equiv s\left(\theta\left(S_{0}, G_{0}\right)\right)$ for some $s \in S_{0}$. Let $x \in A$ with $r(x)=n$. Then $x=f\left(s_{1}, s_{2}, \ldots, s_{m}\right)$, where $s_{1}, s_{2}, \ldots, s_{m} \in$ $G_{n-1}$ and $f \in F$. Hence, there exist $s_{1}^{\prime}, s_{2}^{\prime}, \ldots, s_{m}^{\prime} \in S_{0}$ such that $s_{i} \equiv s_{i}^{\prime}\left(\theta^{\prime}\right)$. Therefore

$$
f\left(s_{1}, s_{2}, \ldots, s_{m}\right) \equiv f\left(s_{1}^{\prime}, s_{2}^{\prime}, \ldots, s_{m}^{\prime}\right)\left(\theta^{\prime}\right) .
$$

On the other hand, $f\left(s_{1}^{\prime}, s_{2}^{\prime}, \ldots, s_{m}^{\prime}\right) \equiv s\left(\theta\left(S_{0}, S_{1}\right)\right)$ for some $s \in S_{0}$. In particular, $x \equiv s\left(\theta^{\prime}\right)$.

COROLlaRY 2. Let $\mathfrak{A}$ be a finitely generated algebra of finite type. If $\mathfrak{A}$ is infinite then there is a congruence relation $\theta$ of $\mathfrak{A}$ such that $\mathfrak{A} / \theta$ is infinite and $\mathfrak{U} / \boldsymbol{\theta}^{\prime}$ is finite for every $\boldsymbol{\theta}^{\prime}>\boldsymbol{\theta}$ in $\operatorname{Con}(\mathfrak{U})$.

Proof. In fact, somewhat more is true: If $\theta_{0} \in \operatorname{Con}(\mathfrak{A})$ with $\mathfrak{A} / \boldsymbol{\theta}_{0}$ finite then there is $\theta \in \operatorname{Con}(\mathfrak{A}), \theta<\theta_{0}$, such that $\mathfrak{A} / \boldsymbol{\theta}$ is infinite and $\mathfrak{A} / \boldsymbol{\theta}^{\prime}$ is finite for every $\theta_{0} \geqslant \theta^{\prime}>\theta$ in Con( $(\mathfrak{U})$. Let $\mathbb{E}$ be a maximal chain of elements $\psi$ of $\operatorname{Con}(\mathfrak{A})$ such that $\mathfrak{U} / \psi$ is infinite and $\psi<\theta_{0}$. According to Theorem 1 , $\bigvee \mathfrak{E}$ would be compact if $\mathfrak{A} / \mathfrak{V}$ were finite; hence, $\mathfrak{A} / \bigvee \mathfrak{E}$ is infinite. Set $\theta=\bigvee \mathfrak{r}$.

Let $\mathfrak{A}$ be an infinite, finitely generated algebra of finite type. If $\theta_{1}, \theta_{2} \in$ $\operatorname{Con}(\mathfrak{U})$ such that $\mathfrak{A} / \boldsymbol{\theta}_{1}, \mathfrak{A} / \boldsymbol{\theta}_{2}$ are finite then $\mathfrak{U} / \boldsymbol{\theta}_{1} \wedge \boldsymbol{\theta}_{2}$ is finite. Now, let $\theta$ be the distinguished member of $\operatorname{Con}(\mathfrak{A})$ guaranteed to exist by Corollary 2 and let $\mathcal{L}$ be any finite family of congruence relations each greater than $\theta$.

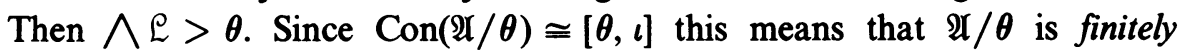
subdirectly irreducible.

COROLlARY 3. Every non-locally-finite variety of finite type contains an infinite finitely subdirectly irreducible member.

We conclude this note with some examples and remarks. While the results above hold for any algebraic system we shall confine our remarks primarily to lattices and groups.

1. The lattices illustrated in Figure 1 are finitely generated; their common congruence lattice is illustrated in Figure 2. Observe that every proper 

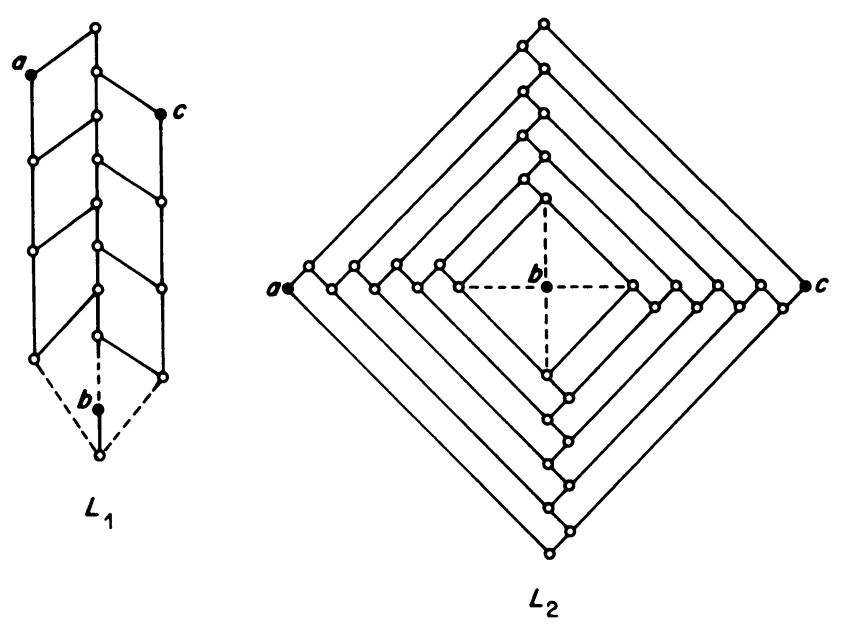

FIGURE 1

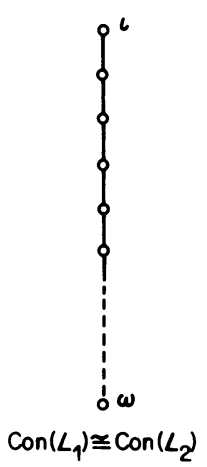

FIGURE 2

quotient of either lattice is finite and every element of its congruence lattice is compact.

2. The hypothesis in Theorem 1 that $\mathfrak{A}$ be finitely generated cannot be dropped. This can be seen by examining the congruence lattice (Figure 3 ) of the lattice illustrated in Figure 4 (cf. [2]).

3. Theorem 1 also serves to highlight the fact that every nontrivial finitely generated algebra has a simple homomorphic image (cf. [3]). For lattices even more is true; namely, any nontrivial lattice $L$ with least element 0 and greatest element 1 has a simple homomorphic image. This follows from the fact that $\operatorname{Con}(L)$ is compactly generated and its maximum element $\iota=$ $\theta(0,1)$ is compact in $\operatorname{Con}(L)$. (Recall that a compact element of a lattice has a lower cover.)

4. An algebra $\mathfrak{A}$ is pseudo-simple if $|\mathfrak{X}|>1$ and $\mathfrak{A} / \theta \cong \mathfrak{A}$ for each $\theta \in$ Con( $(\mathfrak{U})$ satisfying $\theta<\imath$. For instance, the lattice illustrated in Figure 4 is pseudo-simple yet it is not simple. In [4] D. Monk has shown that for every 


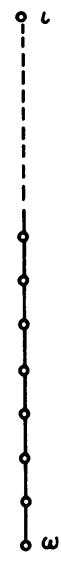

FIGURE 3

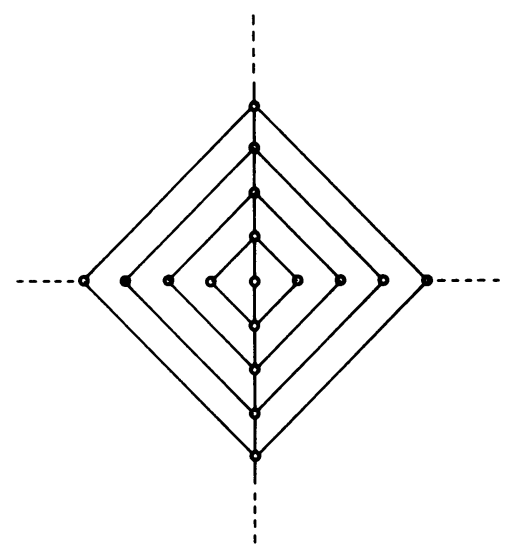

Figure 4

nontrivial pseudo-simple algebra $\mathfrak{A}$, Con( $(\mathfrak{X})$ is a chain of the form $\omega^{\beta}+1$ for some ordinal $\beta$. It now follows that a finitely generated algebra is pseudosimple if and only if it is simple.

5. Theorem 1 provides us with an atomicity condition for the congruence lattice of a finitely generated lattice $L$; that is, every $\theta \in \operatorname{Con}(L)$ with $L / \theta$ finite has a lower cover in $\operatorname{Con}(L)$. Still, not every $\theta \in \operatorname{Con}(L)$ need have a lower cover. Indeed, R. Freese has communicated to us an example of a finitely generated lattice with congruence lattice isomorphic to $\omega+2$.

6. K. Baker has communicated to us the observation that the Theorem generalizes this well-known fact from group theory: In a finitely generated group $G$, a subgroup of finite index must itself be finitely generated. Indeed, let $F$ be the set of left translations on $G$ determined by the designated generators and their inverses, and consider the algebra $\mathbb{S}^{\prime}=\langle G, F\rangle$. In $\mathbb{S S}^{\prime}$, congruence relations correspond to subgroups of $G$, and compact congruence relations to finitely generated subgroups. Theorem 1 can then be applied.

7. Whether or not every variety of lattices that contains only finitely many subvarieties is itself generated by a finite lattice is a question about which very little is known. To settle this question in the affirmative, it suffices to show that every infinite, finitely generated subdirectly irreducible lattice $L$ generates a variety containing infinitely many subvarieties. According to Corollary 2 we may suppose that every proper homomorphic image of $L$ is finite.

For example, it is easy to verify that each of the lattices $L_{1}$ and $L_{2}$ (see Figure 1) has infinitely many nonisomorphic, subdirectly irreducible, homomorphic images; whence each generates a variety with infinitely many subvarieties.

8. It is well known that the congruence lattice of a lattice is a distributive, algebraic lattice. The converse is a long-standing conjecture of lattice theory. In any case, even the congruence lattices of all lattices with least and greatest 
elements cannot account for all distributive algebraic lattices. For instance, the lattice of Figure 3 cannot be the congruence lattice of a lattice with least and greatest elements although it is the congruence lattice of a lattice, namely, of the lattice in Figure 4. We pose one last question: Is every distributive algebraic lattice, whose maximum element is compact and which has only countably many compact elements, the congruence lattice of a finitely generated lattice?

\section{REFERENCES}

1. G. Grätzer, Universal algebra, Van Nostrand, Princeton, N. J., 1968.

2. G. Grätzer and E. T. Schmidt, Two notes on lattice congruences, Ann. Univ. Sci. Budapest. Eötvös Sect. Math. 1 (1958), 83-87.

3. B. Jónsson, Topics in universal algebra, Lecture Notes in Math., vol. 250, Springer-Verlag, Berlin and New York, 1972.

4. D. Monk, On pseudo-simple algebras, Proc. Amer. Math. Soc. 13 (1962), 543-546.

Department of Mathematics, University of Calgary, Calgary, Canada

Department of Mathematics, University of Manitoba, Winnipeg, Canada 